\title{
Gene expression profiles in BCL11B-siRNA treated malignant T cells
}

\author{
Xin Huang ${ }^{1,2 \dagger}{ }^{+}$Qi Shen ${ }^{1 \dagger}$, Si Chen ${ }^{1}$, Shaohua Chen ${ }^{1}$, Lijian Yang ${ }^{1}$, Jianyu Weng ${ }^{2}$, Xin Du², Piotr Grabarczyk ${ }^{3}$, \\ Grzegorz K Przybylski ${ }^{3,4}$, Christian A Schmidt ${ }^{3}$ and Yangqiu Li ${ }^{1,5^{*}}$
}

\begin{abstract}
Background: Downregulation of the B-cell chronic lymphocytic leukemia (CLL)/lymphoma11B (BCL11B) gene by small interfering RNA (siRNA) leads to growth inhibition and apoptosis of the human T-cell acute lymphoblastic leukemia (T-ALL) cell line Molt-4. To further characterize the molecular mechanism, a global gene expression profile of $B C L 11 B$-siRNA -treated Molt-4 cells was established. The expression profiles of several genes were further validated in the BCL11B-siRNA -treated Molt-4 cells and primary T-ALL cells.

Results: 142 genes were found to be upregulated and 109 genes downregulated in the BCL11B-siRNA -treated Molt-4 cells by microarray analysis. Among apoptosis-related genes, three pro-apoptotic genes, TNFSF10, BIK, BNIP3, were upregulated and one anti-apoptotic gene, BCL2L1 was downregulated. Moreover, the expression of SPP1 and CREBBP genes involved in the transforming growth factor (TGF- $\beta$ ) pathway was down 16-fold. Expression levels of TNFSF10, BCL2L1, SPP1, and CREBBP were also examined by real-time PCR. A similar expression pattern of TNFSF10, $B C L 2 L 1$, and SPP1 was identified. However, CREBBP was not downregulated in the BLC11B-siRNA -treated Molt-4 cells.
\end{abstract}

Conclusion: BCL11B-siRNA treatment altered expression profiles of TNFSF10, BCL2L1, and SPP1 in both Molt-4 T cell line and primary T-ALL cells.

\section{Background}

Although treatment outcome in patients with T-cell acute lymphoblastic leukemia (T-ALL) has improved in recent years, relapsed T-ALL remains a challenge [1]. Monoclonal antibodies, gene inhibitors, and upregulation of microRNAs [2,3] are promising tools for cancer targeted therapy. However, few targeted therapies are available for T-cell malignancies. For example, transforming Mer signals may contribute to T-cell leukemogenesis, and regulation of $\mathrm{Mer}$ expression could be a novel therapeutic target for pediatric ALL therapy [4]. The recent identification of activating Notch1 mutations in the majority of patients with T-ALL has brought interests on targeting the Notch signaling pathway for this disease [5].

The B-cell chronic lymphocytic leukemia (CLL)/lymphoma 11B $(B C L 11 B)$ gene was first identified on

\footnotetext{
* Correspondence: yangqiuli@hotmail.com

† Contributed equally

'Institute of Hematology, Medical College, Jinan University, Guangzhou, PR China

Full list of author information is available at the end of the article
}

human chromosome 14q32.2 [6] and encodes a Krüppel-like $\mathrm{C}_{2} \mathrm{H}_{2}$ zinc finger protein initially identified as a transcriptional repressor [7]. BCL11B plays an important role in T-cell differentiation and proliferation [8-11]. Altered expression, mutation, disruption, or rearrangement of $B C L 11 B$ has been associated with T-cell malignancies [12-14]. In humans, $B C L 11 B$ overexpression is found primarily in lymphoproliferative disorders, such as T-ALL and adult T-cell leukemia/lymphoma [12,15-17]. BCL11B mediates transcriptional activation by interacting with the p300 co-activator at the upstream site 1 (US1) of the interleukin (IL)-2 promoter, leading to transcriptional activation of IL-2 expression in activated $\mathrm{T}$ cells [18]. Although the interaction partners and binding sequence have been revealed, only a few $B C L 11 B$ direct target genes have been identified to date. Our previous study in the human T-ALL cell lines Molt-4, Jurkat, and hut78 has shown increased apoptosis upon BCL11B suppression by RNA interference [19]. 
In the present study, we further analyzed the global gene expression profiles in Molt-4 and primary T -ALL cells after $B C L 11 B$-935-siRNA treatment.

\section{Methods \\ Samples}

Samples from three newly diagnosed patients with TALL and one patient with T-cell lymphoma/leukemia were obtained after informed consent. The diagnosis of T-ALL was based on cytomorphology, immunohistochemistry, and flowcytometry analyses. The samples were named P1 (55-year-old male with T-ALL), P2 (6-year-old male with T-ALL), P3 (55-year-old female with T-cell lymphoma/leukemia), and P4 (19-year-old male with TALL). Peripheral blood was collected with heparin and peripheral mononuclear cells (PBMCs; contained more than $70 \%$ leukemic $\mathrm{T}$ cells) were separated using the Ficoll-Hypaque gradient centrifugation method. All procedures were conducted in strict accordance with the guidelines of the Medical Ethics committees of the Health Bureau of Guangdong province, China.

\section{Cell culture}

Molt-4 cells (Institutes for Biological Sciences Cell Resource Center, Chinese Academy of Sciences, Shanghai, China) and PBMCs collected from the four patients were cultured in complete RPMI 1640 medium with $15 \%$ fetal calf serum and were maintained in a sterile incubator at $37^{\circ} \mathrm{C}, 95 \%$ humidity, and $5 \% \mathrm{CO}_{2}$.

\section{Nucleofection}

$B C L 11 B$-siRNA935 (Chinese patent application number: 200910193248.3) and the scrambled non-silencing siRNA control (BCL11B-sc) were designed with online software http://www.invitrogen.com and synthesized by Invitrogen (Carlsbad, CA, USA).

Malignant T cells were resuspended at $2.5 \times 10^{6}$ (Molt-4 cells) or $1 \times 10^{7}$ (PBMCs) per $100 \mu \mathrm{L}$ of the appropriate Nucleofector kit solution (Amaxa Biosystems, Cologne, Germany), and were nucleofected with $3 \mu \mathrm{g}$ of BCL11BsiRNA or control non-silencing scrambled (sc) RNA using the C-005 (Molt-4 cells) or U-014 (PBMCs) program in the Nucleofection Device II (Amaxa Biosystems). Mocktransfected cells (nucleofected without siRNA) were used as a negative control. After nucleofection, the cells were immediately mixed with $500 \mu \mathrm{L}$ of pre-warmed culture medium and transferred to culture plates for incubation. Samples were collected for RNA isolation.

RNA isolation, expression profiling, reverse transcription, and real-time PCR

Total RNA was isolated using Trizol (Invitrogen), and cDNA was synthesized with a Superscript II RNaseH Reverse Transcriptase kit (Invitrogen).
Total RNA ( $>3 \mu \mathrm{g}$ ) was sent for global gene expression profile analysis using an Affymetrix HG U133 Plus 2.0 gene chip (Shanghai Biochip Co., Ltd., Shanghai, China). The Affymetrix microarray analysis was performed using Gene Spring GX10.0 software (Agilent Technologies, Santa Clara, CA, USA).

The primer and probe information for $B C L 11 B$ and the reference gene $\beta$-2-microglobulin $(\beta 2-M G)$, as well as the details of the real-time PCR for $B C L 11 B$ were described in our previous studies [12,15]. Expression levels of tumor necrosis factor (ligand) superfamily, member 10 (TNFSF10; TRAIL), BCL2-like 1 (BCL2L1; $B c l-x L)$, secreted phosphoprotein 1 (SPP1), cAMPresponse element binding protein (CREBBP), and $\beta 2$ $M G$ were determined by real-time PCR using a SYBR Green I qPCR Master Mix kit [15].

\section{Flow cytometry assay}

Cells from different groups were prepared according to the protocols, and the $B C L 2$ expression level was measured by flow cytometry (Beckman Coulter, Fullerton, CA, USA). Mouse anti-human BCL2-PE and mouse IgG1-PE (eBioscience, San Diego, CA, USA) were used. Results were analyzed using the Win MDI 2.9 software.

\section{Results and discussion}

\section{Global gene expression profile in BCL11B-siRNA935} treated Molt-4 cells

To determine the molecular mechanisms of $B C L 11 B$ siRNA-mediated cell apoptosis, global gene expression profiling was performed at $24 \mathrm{~h}$ post-transfection, when $B C L 11 B$ mRNA was most effectively suppressed (data not shown). Results were clustered, based on the differential expression level (2-fold up or down), and visualized using a color scale (Figure 1A). Principal component analysis indicated that the changes in the Molt-4 cell gene expression profile could be accounted for primarily by the $B C L 11 B$ siRNA935 treatment (Figure 1B). A GCOS1.4 software analysis showed that upregulated genes were identified by 142 probe sets, whereas 109 genes were downregulated at least 2-fold, compared with the sc control (Figure 1C). Changes in genes of the same signaling pathways closely related to tumor cell proliferation and apoptosis were analyzed further (Figure 1D).

Among apoptosis-related genes, changes in expression levels occurred mainly in three pro-apoptotic genes; TNFSF10, $B C L-2$ interacting killer $(B I K)$, and $B C L-2 /$ $E 1 B 19 \mathrm{kDa}$ interacting protein 3 (BNIP3), which were upregulated 2-4 fold, and one anti-apoptotic gene (BCL2L1) was downregulated by 3-4 fold. The expression levels of SPP1 and CREBBP genes involved in the transforming growth factor (TGF- $\beta$ ) pathway were down by 16 fold. The changes in the expression levels of the 


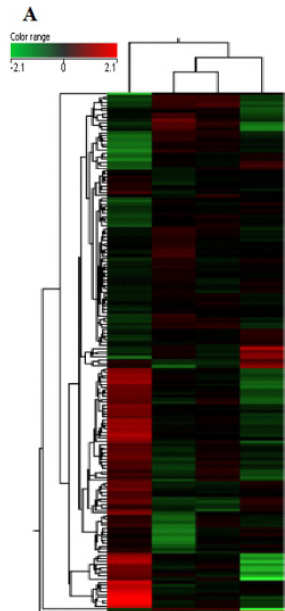

SiRNA935 SC MOCK NC

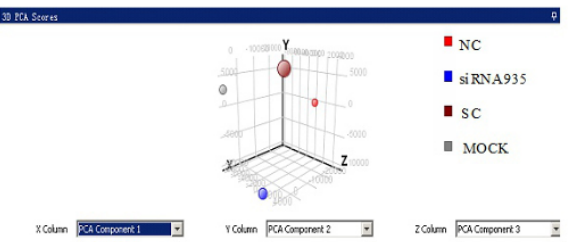

C

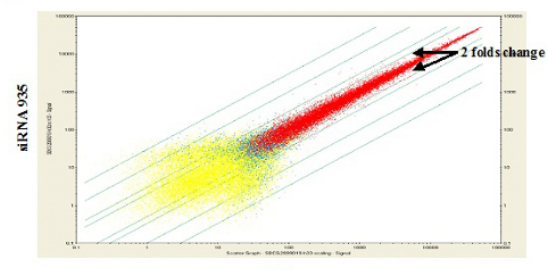

SC

D

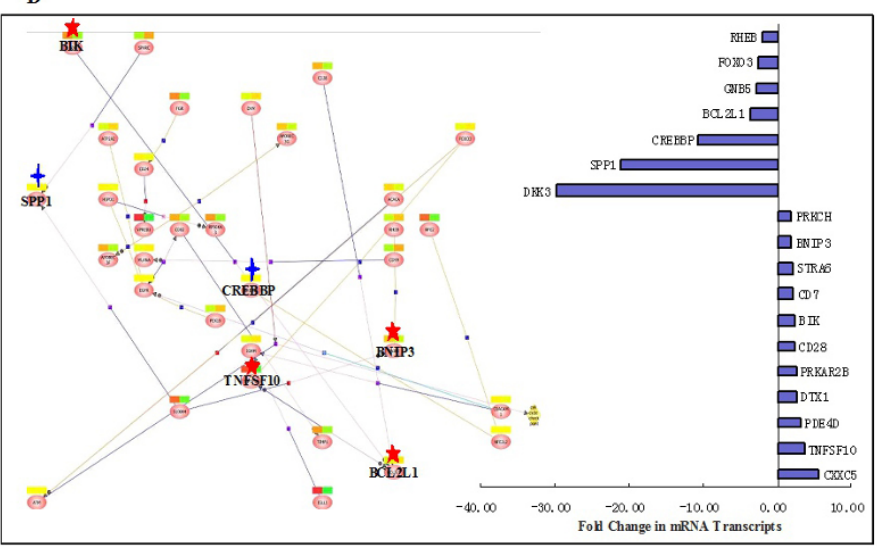

$\mathbf{F}$
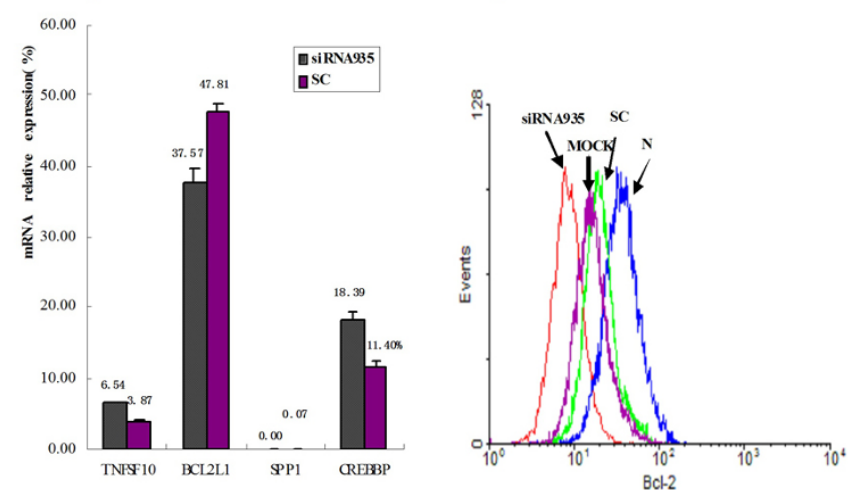

Figure 1 Results of the gene chip microarray analysis and validation. (A) Visual display of the cluster analysis for the $B C L 11 B$ siRNA935transfected and control cells. (B) Principal component analysis. The closer the dots, the more similar the gene expression profiles are; the farther apart the dots, the greater the differences are. (C) Two-dimensional scatterplot analysis of gene expression values for all genes on the BCL $11 B$ siRNA935-transfected cells and control cells from the microarray. Yellow dots represent genes absent from both samples; blue dots represent genes present in one sample but absent from the other sample; and red dots represent genes present in both samples. Dots outside the $2 \times$ difference lines, indicated by black arrows, represent differentially expressed genes. The farther from the line, the greater the difference in gene expression are. (D) Analysis of pathways closely related to tumor cell proliferation and apoptosis. Results are shown as fold-change in mRNA transcripts. Genes indicated with a red star are in the apoptosis pathway; genes indicated with a blue star are in the transforming growth factor$\beta$ pathway. (E) Gene validation by real-time PCR. Changes in TNFSF10, BCL2L1, and SPP1 expression levels agreed with the microarray results, while those of CREBBP did not. (F) Reduced BCL-2 protein expression was confirmed by flow cytometry. BCL-2 expression in BCL11B siRNA3transfected cells was significantly lower, at 46\% of that in SC (99.1\%), MOCK (99.2\%), and NC cells (99.7\%). 
TNFSF10, BCL2L1, SPP1, and CREBBP genes were further detected by real-time PCR (Figure 1E). The BH3-only domain proteins BIK and BNIP3, which were located upstream of BCL-2 (Figure 2), may enhance their binding to BCL-2, thereby inhibiting the anti-apoptotic function. Thus, we analyzed the $B C L-2$ protein expression level by flow cytometry in Molt-4 cells at 72 $\mathrm{h}$ after $B C L 11 B$-siRNA treatment (Figure 1F). A similar altered expression pattern of these genes, as well as expression of the $B C L-2$ protein, was confirmed. However, CREBBP did not show downregulation in BCL11BsiRNA treated Molt- 4 cells.

The global gene expression profile results suggest that the molecular mechanisms of $B C L 11 B$ siRNA-mediated cell death may involve $B C L-2$ family genes in the intrinsic mitochondrial pathway as well as the TNFSF 10 gene in the death receptor signaling pathway (Figure 2) [20]. Upregulation of the TNFSF10 gene activated the death receptor signaling pathway, whereas upregulation of the two mitochondrial $B C L-2$ family genes (the BH3-only domain proteins $B I K$ and $B N I P 3$ ) enhanced their binding to $B C L-2$, with a reduction in the anti-apoptotic gene $B C L 2 L 1$, thereby inhibiting the anti-apoptotic function and promoting Bax and Bak activation. This in turn activates the downstream caspases 3,6 , and 7 , leading to increased apoptosis. Reduced expression of SPP1 correlated with increased apoptosis in Molt-4 cells, suggesting that the $S P P 1$ gene may be a $B C L 11 B$ gene target.

$C R E B B P$ overexpression has been detected in Jurkat cells [21]. However, previous studies have not reported a change in CREBBP expression in $\mathrm{T}$ cell lines after BCL11B-siRNA treatment. In the present study, downregulation of $C R E B B P$ was identified in the microarray analysis, but not confirmed by real-time PCR analysis. The reason may be due to a systemic error on the microarray analysis. Interestingly, unlike the result from Molt- 4 cells, the alteration of the CREBBP expression level in primary T-All cells after BCL11B-siRNA treatment was in accordance with the results from the microarray analysis (Figure 3 ). Thus, the role of $C R E B B P$ during $B C L 11 B$ downregulation in malignant $\mathrm{T}$ cells requires further investigation.

Expression of TNFSF10, BCL2L1, SPP1, and CREBBP genes in $B C L 11 B$-siRNA935-treated primary leukemic $T$ cells After obtaining interesting data from Molt-4 cells, we analyzed the effect of the $B C L 11 B$-siRNA in primary $\mathrm{T}$ ALL cells. We examined the expression levels of TNFSF10, BCL2L1, SPP1, and CREBBP in primary leukemic T cells after $B C L 11 B$ siRNA935 treatment.

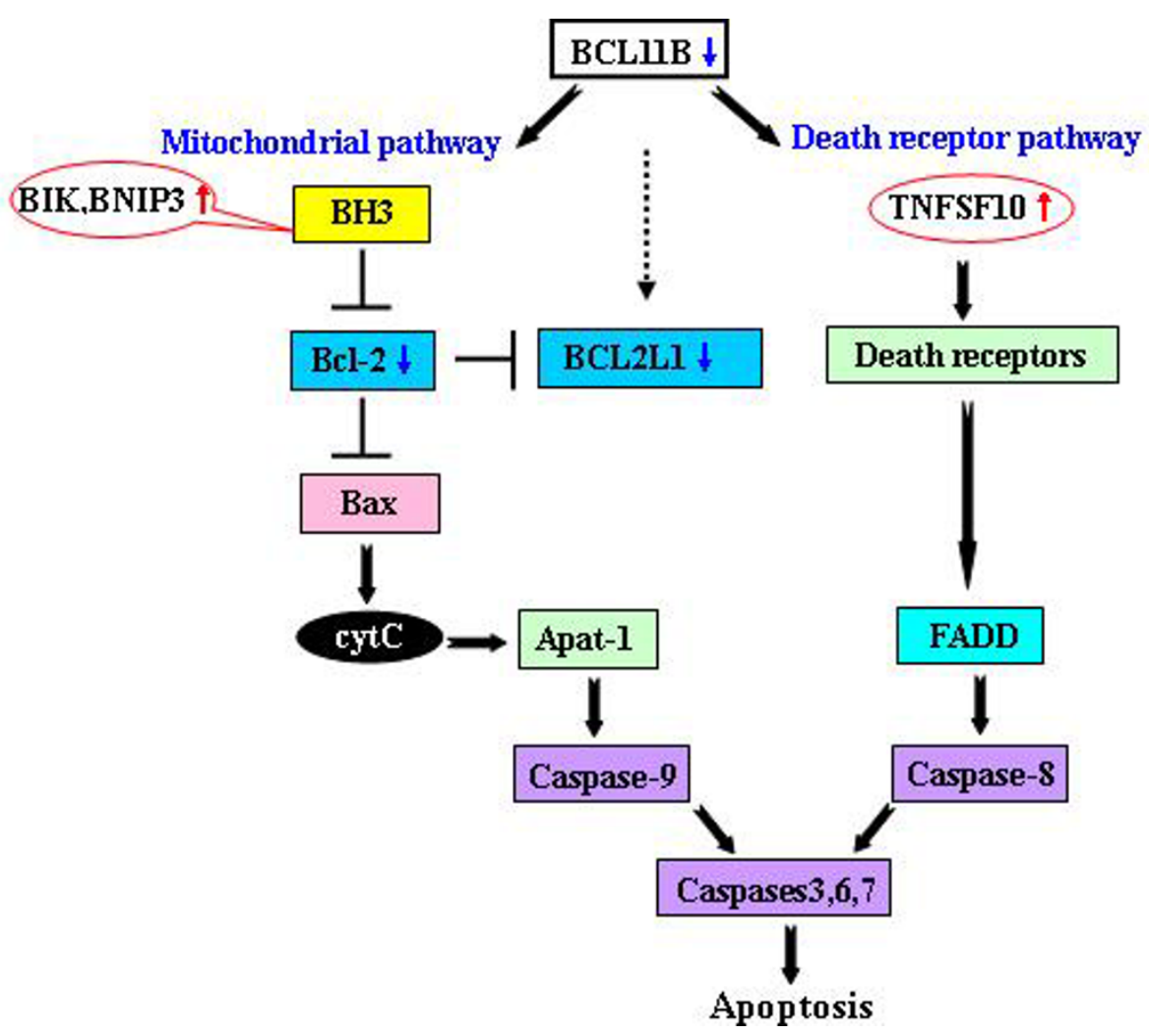

Figure 2 Schematic model of the molecular mechanism of BCL11B-siRNA-mediated apoptosis in Molt-4 cell [modified from reference 20] 


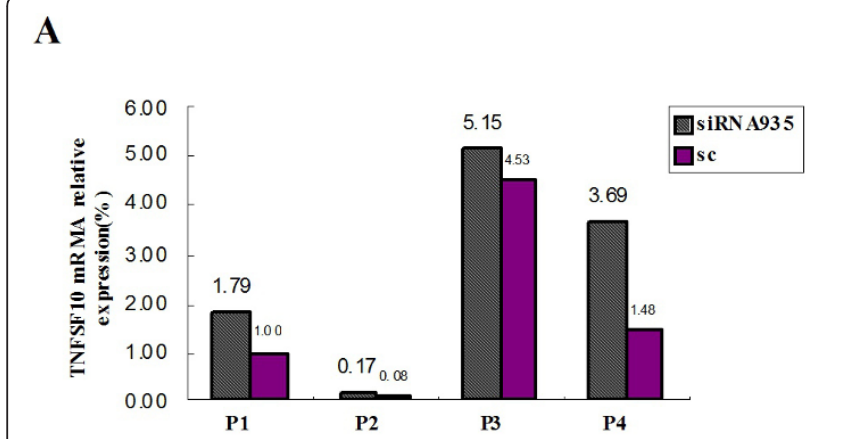

B

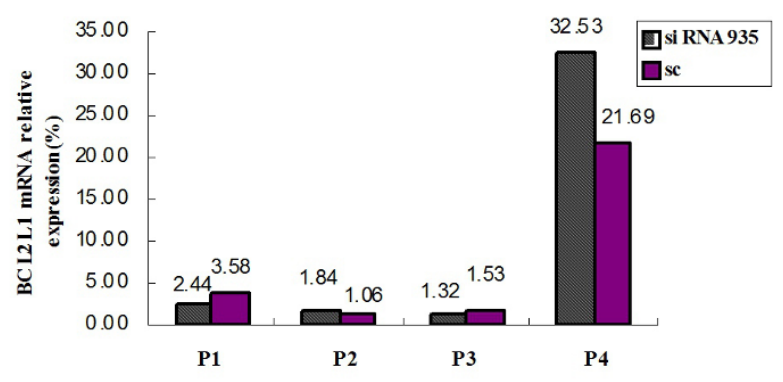

C

D
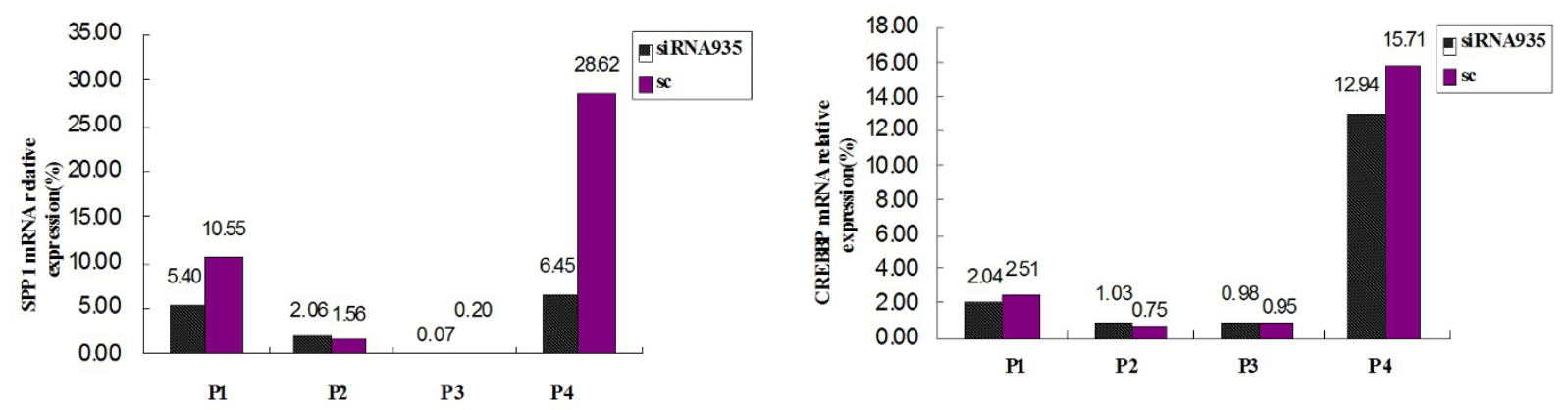

Figure 3 Expression of TNFSF10, BCL2L1, SPP1, and CREBBP genes in peripheral mononuclear cells from four patients (P1-P4) with Tcell acute lymphoblastic leukemia at $24 \mathrm{~h}$ after $B C L 11 B$ siRNA transfection.

$B C L 11 B$ expression level decreased in primary leukemic T cells treated with $B C L 11 B$ siRNA935 (282.77 \pm 247.57 copies $\left./ 10^{5} \beta 2-M G\right)$ as compared with the sc control group $\left(519.48 \pm 303.41\right.$ copies $\left./ 10^{5} \quad \beta 2-M G\right)$. The TNFSF10, BCL2L1, SPP1, and CREBBP expression levels in $B C L 11 B$-siRNA935- treated primary leukemic $\mathrm{T}$ cells were $2.7 \pm 2.17 \%, 9.53 \pm 15.34 \%, 3.5 \pm 2.95 \%$, and 4.25 $\pm 5.82 \%$, respectively, whereas the expression levels in primary leukemic $\mathrm{T}$ cells in the sc control group were $1.77 \pm 1.93 \%, 6.96 \pm 9.88 \%, 10.23 \pm 13.09 \%$, and $4.98 \pm$ $7.2 \%$, respectively. The T-ALL specimen number was too small to perform statistical analysis. The changes in the mRNA levels of TNFSF10, SPP1, and CREBBP in the $\mathrm{T}$ cells from the four patients agreed in general with those from the microarray analysis results (Figure 3A, C, D). However, the changes in the BCL2L1 expression levels in the different samples varied (Figure $3 B$ ). The reduced $B C L 2 L 1$ expression rates in leukemic $\mathrm{T}$ cells from patients 1 and 3 were $31.84 \%$ and $13.73 \%$, respectively, compared with the sc controls, whereas BCL2L1 expression in leukemic $\mathrm{T}$ cells from patients 2 and 4 was upregulated. Although $B C L 11 B$ gene overexpression occurred in all samples, it may have been due to the heterogeneity of $\mathrm{T}$-cell malignancies during apoptosis induced by $B C L 11 B$ downregulation [22], so it remains to be determined whether apoptosis induced by $B C L 11 B$ downregulation in some cases with T-ALL involves the $B C L-2$ family genes in the intrinsic mitochondrial pathway.

A previous analysis revealed that overexpression of the $B C L 11 B, B C L 2 L 1$, and CREBBP genes in primary T-ALL samples blocks apoptosis in malignant T cells [15]. This study suggests that inhibition of $B C L 11 B$ may trigger apoptosis in leukemic $\mathrm{T}$ cells by downregulating the downstream genes SPP1, CREBBP, and TNFSF 10 .

\section{Conclusions}

Our findings provide evidence that BCL11B siRNAmediated cell apoptosis may be related to the mitochondrial pathway $B C L-2$ family genes and the TNFSF 10 gene of the death receptor signaling pathway. Moreover, the SPP 1 and CREBBP genes in the TGF- $\beta$ pathway may also be involved in $B C L 11 B$ siRNA-mediated cell apoptosis. 


\section{Acknowledgements}

The authors thank Dr. Xuesong Yang for critical reading of this manuscript, and thank Dr. Xuchao Zhang from the Cancer Center of Guangdong Provincial Hospital for helpful analysis of the gene-chip data. This work was supported by Grants from National Natural Science Foundation of China (no. 30771980) and the Guangdong Science \& Technology Project (no. 2007B030703008; and 2009B050700029).

\section{Author details}

${ }^{1}$ Institute of Hematology, Medical College, Jinan University, Guangzhou, PR China. ${ }^{2}$ Department of Hematology, Guangdong General Hospital (Guangdong Academy of Medical Sciences), Guangzhou, PR China. ${ }^{3}$ Department of Hematology and Oncology, Ernst-Moritz-Arndt University Greifswald, Greifswald, Germany. ${ }^{4}$ Institute of Human Genetics, Polish Academy of Sciences, Poznan, Poland. ${ }^{5}$ Key Laboratory for Regenerative Medicine of Ministry of Education, Jinan University, Guangzhou, PR China.

\section{Authors' contributions}

YQL contributed to concept development and study design. XH, QS, SC, SHC, LY performed the laboratory studies. PG, GKP and CAS provided some materials and technical support. JYW and XD were responsible for collection of clinical data. YQL and XH coordinated the study and helped drafting the manuscript. All authors read and approved the final manuscript.

\section{Competing interests}

The authors declare that they have no competing interests.

Received: 21 March 2011 Accepted: 15 May 2011

Published: 15 May 2011

\section{References}

1. Aifantis I, Raetz E, Buonamici S: Molecular pathogenesis of T-cell leukaemia and lymphoma. Nat Rev Immunol 2008, 8:380-390.

2. Budhu BA, Ji JF, Wang XW: The clinical potential of microRNAs. J Hematol Oncol 2010, 3:37.

3. Wei GQ, Rafiyath S, Liu DL: First-line treatment for chronic myeloid leukemia:dasatinib, nilotinib, or imatinib. J Hematol Oncol 2010, 3:47.

4. Graham DK, Salzberg DB, Kurtzberg J: Ectopic expression of the protooncogene Mer in pediatric T-cell acute lymphoblastic leukemia. Clin Cancer Res 2006, 12:2662-2669.

5. Palomero T, Ferrando A: Therapeutic targeting of NOTCH1 signaling in TALL. Clin Lymphoma Myeloma 2009, 9(Suppl 3):S205.

6. Satterwhite E, Sonoki T, Willis TG, Harder L, Nowak R, Arriola EL, Liu H, Price HP, Gesk S, Steinemann D, Schlegelberger B, Oscier DG, Siebert R, Tucker PW, Dyer MJ: The BCL11 gene family: involvement of BCL11A in lymphoid malignancies. Blood 2001, 98:3413-3420.

7. Avram D, Fields A, Senawong T, Topark-Ngarm A, Leid M: COUP-TF (chicken ovalbumin upstream promoter transcription factor)-interacting protein 1 (CTIP1) is a sequence-specific DNA binding protein. Biochem J 2002, 368:555-563.

8. Li L, Leid M, Rothenberg EV: An Early T cell lineage commitment checkpoint dependent on the transcription factor Bcl11b. Science 2010, 329:89-93.

9. Wakabayashi Y, Watanabe H, Inoue J, Takeda N, Sakata J, Takeda N, Sakata J, Mishima Y, Hitomi J, Yamamoto T, Utsuyama M, Niwa O, Aizawa S, Kominami $\mathrm{R}$ : $\mathrm{Bcl} 11 \mathrm{~b}$ is required for differentiation and survival of $a \beta T$ lymphocytes. Nat Immunol 2003, 4:533-539.

10. Cismasiu VB, Ghanta S, Duque J, Albu D, Chen HM, Kasturi R: BCL11B participates in the activation of interleukin-2 gene expression in CD4+ $T$ lymphocytes. Blood 2006, 108:2695-2702.

11. Liu P, Li P, Burke S: Critical roles of Bcl11b in T-cell development and maintenance of T-cell identity. Immunol Rev 2010, 238:138-149.

12. Przybylski GK, Dik WA, Wanzeck J, Grabarczyk P, Majunke S, MartinSubero Jl, Siebert R, Dölken G, Ludwig WD, Verhaaf B, van Dongen J J, Schmidt CA, Langerak AW: Disruption of the BCL11B gene through inv 14 q11.2q32.31 results in the expression of BCL11B-TRDC fusion transcripts and is associated with the absence of wild-type BCL11B transcripts in TALL. Leukemia 2005, 19:201-208.

13. Karlsson A, Nordigården A, Jönsson Jl, Söderkvist P: Bcl11b mutations identified in murine lymphomas increase the proliferation rate of hematopoietic progenitor cells. BMC Cancer 2007, 7:195.
14. Su XY, Della-Valle V, Andre-Schmutz I, Lemercier C, Radford-Weiss I, Ballerini $P$, Lessard M, Lafage-Pochitaloff M, Mugneret F, Berger R, Romana SP, Bernard OA, Penard-Lacronique V: HOX11L2/TLX3 is transcriptionally activated through $\mathrm{T}$-cell regulatory elements downstream of BCL11B as a result of the $t(5 ; 14)$ (q35;q32). Blood 2006, 108:4198-4201.

15. Huang X, Chen S, Shen Q, Yang L, Li B, Zhong LY, Geng SX, Du X, Li YQ: Analysis of the expression pattern of the BCL11B gene and its relatives in patients with T-cell acute lymphoblastic leukemia. $J$ Hematol Oncol 2010, 3:44.

16. Nagel S, Kaufmann M, Drexler HG, MacLeod RA: The cardiac homeobox gene NKX2-5 is deregulated by juxtaposition with BCL11B in pediatric TALL cell lines via a novel $t(5 ; 14)(q 35.1 ; q 32.2)$. Cancer Res 2003, 63:5329-5334

17. Oshiro A, Tagawa H, Ohshima K, Karube K, Uike N, Tashiro Y, Utsunomiya A, Masuda M, Takasu N, Nakamura S, Morishima Y, Seto M: Identification of subtype-specific genomic alterations in aggressive adult T-cell leukemia/ lymphoma. Blood 2006, 107:4500-4507.

18. Cismasiu VB, Ghanta S, Duque J, Albu DI, Chen HM: BCL11B participates in the activation of IL2 gene expression in CD4+ T lymphocytes. Blood 2006, 108:2695-2702

19. Huang $X$, Chen S, Chen SH, Yang LJ, Shen Q, Grabarczyk P, Przybylski GK, Schmidt CA, Li YQ: Inhibition of BCL11B expression leads to apoptosis of malignant T cell lines but not CD34+ cells [abstract]. Blood 2010, 116:1539.

20. Adams JM, Cory S: The Bcl-2 apoptotic switch in cancer development and therapy Bcl-2 apoptotic switch in cancer. Oncogene 2007, 26:1324-1337.

21. Caravatta L, Sancilio S, di Giacomo V, Rana R, Cataldi A, Di Pietro R: PI3-K/ Akt-dependent activation of cAMP-response element-binding (CREB) protein in Jurkat T leukemia cells treated with TRAIL. J Cell Physio/ 2008, 214:192-200.

22. Onciu M, Lai R, Vega F, Bueso-Ramos C, Medeiros LJ: Precursor T-cell acute lymphoblastic leukemia in adults: age-related immunophenotypic, cytogenetic, and molecular subsets. Am J Clin Pathol 2002, 117:252-258.

doi:10.1186/1756-8722-4-23

Cite this article as: Huang et al:: Gene expression profiles in $B C L 11 B$ siRNA treated malignant T cells. Journal of Hematology \& Oncology 2011 $4: 23$.

\section{Submit your next manuscript to BioMed Central and take full advantage of:}

- Convenient online submission

- Thorough peer review

- No space constraints or color figure charges

- Immediate publication on acceptance

- Inclusion in PubMed, CAS, Scopus and Google Scholar

- Research which is freely available for redistribution 